\title{
Using new analytical tools to produce better embryos in vitro
}

\author{
J.R. Herrick, E. Silva, R.L. Krisher ${ }^{1}$
}

Colorado Center for Reproductive Medicine, 10290 RidgeGate Circle, Lone Tree, USA.

\begin{abstract}
The purpose of this review is to summarize what we know about preimplantation embryo metabolism, focusing on ruminant species, and to discuss how this knowledge informs our approach to culturing embryos in vitro. The important relationship between embryo metabolism and viability will be emphasized, and theories of metabolic networks in embryos presented. Methods that have historically been used to study embryo metabolism will be compared and contrasted to a new method of evaluating embryo metabolism; metabolomics. Finally, the advantages and disadvantages of using metabolomics technologies to study embryo metabolism will be critically evaluated. The application of metabolomics to assisted reproductive technologies, and specifically to embryo culture, will be highlighted. We conclude that use of metabolomics to study embryo physiology will enlighten our understanding of embryo metabolic pathways in the context of a complete media that enables good blastocyst production. This way of thinking about embryo metabolism as dynamic, complex and interrelated biochemical pathways, informed by metabolomics, will allow us to develop the next generation of embryo culture medium to support and manipulate metabolism to promote embryo viability, as well as to identify the most viable embryos for transfer.
\end{abstract}

Keywords: embryo, in vitro culture, metabolism, viability.

\section{Introduction}

Embryos can develop successfully to the blastocyst stage in a wide variety of commercially available culture media. Although embryos produced in vitro exhibit only slightly lower pregnancy rates than those produced in vivo, they exhibit reduced survival following vitrification and have multiple associated problems during pregnancy and parturition, including heavier birth weight, extended gestation, and a higher incidence of fetal and neonatal loss, suggesting reduced embryo quality (Hasler, 2000; Rizos et al., 2002). Improvements have been made in the culture of embryos from domestic species in the last decade, but significant progress in optimizing in vitro embryo production remains elusive because we still do not fully understand embryonic metabolism. Preimplantation embryos exhibit an astonishing degree of metabolic plasticity, allowing them to use a variety of metabolic substrates via multiple pathways to support development in a variety of media that often bear little resemblance to the composition of oviductal or uterine fluid. This complicates the determination of optimal nutrient provisions to support development in vitro. Although embryos are capable of adapting their metabolic activity to utilize a variety of nutrients in their environment, the metabolic costs of adaptation to suboptimal culture conditions can compromise embryo viability, cryotolerance, maintenance of pregnancy, fetal growth, and offspring health. This relationship between metabolic activity and viability is central to the successful application of assisted reproductive technologies. Only by understanding the metabolic requirements of the embryo can we design culture systems that support the development of viable embryos with the best chance of resulting in healthy offspring.

The application of metabolomics to the analysis of embryo metabolism is helping to further this understanding. Metabolomics permits investigation of embryo physiology in a focused, in depth manner that has not been previously possible, allowing us to think about embryo metabolism as a complex interplay of multiple metabolic mechanisms. This technology has tremendous potential to expand our knowledge of embryo metabolism because it can be applied noninvasively to the study of embryo physiology via the simultaneous measurement of multiple substrates following culture in an optimized medium. A metabolomics approach not only provides information about suspected pathways of importance, but also about unknown regulatory mechanisms and metabolic intermediates. Information provided by metabolomics will inform the development of improved embryo culture media to reduce in vitro stress and adaptation, as well as methods to regulate metabolism in vitro to improve embryo quality. In addition, specific metabolic fingerprints characteristic of high quality embryos will be discovered.

\section{Metabolic networks in mammalian embryos}

Existing studies have provided a glimpse of the diverse metabolic mechanisms used by embryos, and hinted at the dynamic, tightly controlled biochemistry over the time course of preimplantation development. However, we have only begun to appreciate these mechanisms and how they are controlled. Interpretation of metabolic studies is complicated by in vitro conditions, and we still do not have a good understanding of how embryos are operating metabolically within the larger context of their environment, much less what pathways they should be 
utilizing to promote optimal quality. Several hypotheses, not necessarily mutually exclusive, have been proposed to understand embryo metabolism in a larger context. The Quiet Embryo Hypothesis proposes that viable embryos have lower overall metabolism because they are not responding to cellular stress (Leese, 2002; Baumann et al., 2007; Leese et al., 2007, 2008). Energy requirements are increased with stress, suggesting that elevated metabolism has a negative relationship with embryo viability. Embryo metabolism has also been hypothesized to mirror that of cancer cells, which use a metabolic strategy known as the Warburg Effect (Warburg, 1956; Krisher and Prather, 2012; Smith and Sturmey, 2013). Warburg metabolism is thought to support rapid cellular proliferation by providing precursors for macromolecular synthesis and oxidative stress management, and is known to be involved in the control of cellular differentiation (Vander Heiden et al., 2009, 2010; Panopoulos et al., 2012; Zhang et al., 2012). Although the tricarboxylic acid (TCA) cycle is a much more efficient means of producing ATP when compared to glycolysis, the embryo may have more important metabolic uses for glucose than simply ATP production. This may include redox control and macromolecular synthesis, including DNA, RNA, proteins and lipids to support rapid embryonic growth. It may be too simplistic to view embryo glucose metabolism as primarily a means to produce ATP, ignoring the embryo's biosynthetic requirements. In this case, fatty acids and/or amino acids likely support basal TCA activity to provide ATP.

\section{Embryo metabolism}

Although embryo metabolism has been well investigated, the basis of our current understanding primarily comes from studies of the murine embryo and its use of a limited number of substrates, primarily the carbohydrates glucose, lactate and pyruvate and occasionally the amino acid glutamine. The pioneering studies of Biggers and Brinster (Brinster, 1965a, b; Biggers et al., 1967) led to a model in which cleavage stage embryos primarily utilize pyruvate and lactate while a reliance on glucose metabolism via glycolysis characterizes the blastocyst prior to implantation, when higher glucose uptake is a signature of viability (Gardner and Leese, 1987; Gardner et al., 2001). Fifty years later, this model is still surprisingly accurate and widely cited as the generalized pattern of metabolic activity in the mammalian embryo. However, these studies were conducted in simple media (salts, carbohydrates, and protein) that do not support optimal development. For example, oviductal and uterine fluids contain all 20 of the basic amino acids (Harris et al., 2005; Hugentobler et al., 2007; Li et al., 2007), in contrast to early culture medium formulations in which glucose and lactate were present in supra-physiological concentrations and no amino acids were present (Brinster, 1965a; Whitten and Biggers, 1968).

In ruminants, pyruvate uptake exceeds that of glucose at the early cleavage stages (Rieger et al., 1992; Gardner et al., 1993; Thompson et al., 1996). Similarly, $\sim 90 \%$ of ATP is derived from oxidative metabolism prior to compaction, with pyruvate and glutamine being the preferred substrates (Thompson et al., 1991, 1996; Rieger et al., 1992; Gardner et al., 1993). Even though glucose is not the "preferred substrate", early bovine embryos do utilize glucose, with increases in PPP and glycolysis during preimplantation development (Wales and Brinster, 1968; Leese and Barton, 1984; Pantaleon et al., 2001; Comizzoli et al., 2003). Glucose consumption, hexokinase activity, and lactate production increase from the zygote to morula stages (Wales and Brinster, 1968; Leese and Barton, 1984; Gardner and Leese, 1986, 1988; O'Fallon and Wright, 1986; Saito et al., 1994; Houghton et al., 1996). In postcompaction ruminant embryos there is a shift to glucose metabolism, with an increase in glucose uptake, lactate production, glycolytic activity, and the proportion of ATP produced via glycolysis (Thompson et al., 1991, 1996; Rieger et al., 1992; Gardner et al., 1993). Pyruvate uptake and oxidation also increase during blastocyst development (Rieger et al., 1992; Gardner et al., 1993; Thompson et al., 1993, 1996; Krisher et al., 1999; Khurana and Niemann, 2000), even though glucose is the primary substrate. Oxidation of lactate and pyruvate appear to be inversely related, with inclusion of one substrate in the medium inhibiting metabolism of the other during pre-compaction development (Khurana and Niemann, 2000). Isolated trophectoderm cells from bovine blastocysts consumed less glucose and more pyruvate, and produced more lactate than inner cell mass cells (Gopichandran and Leese, 2003).

Glucose is present in the bovine oviduct at a concentration of $\sim 2.5 \mathrm{mM}$ (Hugentobler et al., 2008, 2010). However, there are culture media that successfully support development of bovine preimplantation embryos with glucose (SOF; Tervit et al., 1972; Steeves and Gardner, 1999; Gandhi et al., 2000) and without glucose (CRlaa; Rosenkrans and First, 1994); (mSOF; Takahashi and First, 1992). Because the cow and pig embryo are able to develop in vitro from the 1-cell stage to blastocyst in the absence of exogenous glucose, without any known detrimental consequences, it may be possible that pyruvate is converted to phosphoenolpyruvate (PEP) by mitochondrial enzymes, which may participate in the reversible reactions of glycolysis to supply intermediates for the PPP.

With the exception of glutamine, the majority of metabolic studies have focused on carbohydrates. However, porcine, bovine, and ovine embryos will develop to the blastocyst stage with protein and/or amino acids (AA) as the only exogenous nutrient sources (Petters et al., 1990; Thompson et al., 1992; Sutton-McDowall et al., 2012). Numerous studies have shown that AA have beneficial effects on the development of embryos from multiple species when added to the culture medium (Liu and Foote, 1995; McKiernan et al., 1995; Lane and Gardner, 1997; Steeves and Gardner, 1999; Biggers et al., 2000; Lane et al., 2001; Suzuki and Yoshioka, 2006). Specific amino 
acids are consumed (depleted from the medium) and produced (secreted into the medium) by embryos from mice, pigs, cattle, and humans (Houghton et al., 2002; Orsi and Leese, 2004; Humpherson et al., 2005; Wale and Gardner, 2012). Presumably some of the consumed amino acids are used for protein synthesis, but other possible fates for the amino acids are not clear. Amino acids can act as osmotic buffers, helping the embryo to maintain cellular homeostasis (Baltz and Zhou, 2012). Ammonium production by embryos cultured with amino acids also indicates that some amino acids are being converted to TCA cycle intermediates for generation of ATP (Gardner et al., 2001; Lane et al., 2001). Since ammonium can be inhibitory to development, embryos have mechanism to detoxify ammonium and prevent its build-up in the cytoplasm or the culture medium. Murine and bovine embryos are capable of producing glutamine from ammonium and glutamate and/or producing alanine from glutamate, pyruvate, and ammonium (Orsi and Leese, 2004; Wale and Gardner, 2013). The resulting alanine and glutamine are secreted into the medium, which has been observed in a number of studies (Houghton et al., 2002; Orsi and Leese, 2004; Humpherson et al., 2005; Wale and Gardner, 2013; Krisher et al., 2015).

The study of carbohydrate metabolism has overshadowed the contribution of fatty acid $\beta$-oxidation (FAO) until relatively recently. Cow, pig and cat oocytes have large stores of intracellular lipids while the mouse has fewer lipid stores, a fact reflected by the color of the cytoplasm (McEvoy et al., 2000; Leroy et al., 2005a). In humans and domestic ruminants, palmitic, stearic and oleic are the most abundant fatty acids in oocytes, while pig oocytes contain greater polyunsaturated fatty acids, particularly linoleic acid (Homa et al., 1986; Matorras et al., 1998; McEvoy et al., 2000; Kim et al., 2001). Even those species with a relatively low concentration of lipids, like mice, rabbits, and humans, have been shown to actively metabolize this nutrient source (Khandoker and Tsujii, 1998; Haggarty et al., 2006; Dunning et al., 2010; Paczkowski et al., 2014). Inhibition of fatty acid oxidation decreases embryonic development in both mice and cattle (Hewitson et al., 1996; Ferguson and Leese, 2006). The addition of fatty acids or carnitine to stimulate FAO to oocyte and embryo culture medium has primarily shown positive effects on development, although results are variable due in part to differences in type and concentration of fatty acid used (Spindler et al., 2000; Leroy et al., 2005b; Dunning et al., 2010; Marei et al., 2010; Somfai et al., 2011; Van Hoeck et al., 2011; Wu et al., 2011).

\section{Historic approaches to measuring metabolism}

To date, most of what we know about embryo metabolism has been determined using radiolabeled substrates or microfluorescence. Radiolabeled substrates provide information about specific pathways, depending on the location of the label on the original substrate and the end metabolite. Microfluorescence is based upon enzymatically coupled reactions associated with changing ratios of $\mathrm{NAD}(\mathrm{P})+: \mathrm{NAD}(\mathrm{P}) \mathrm{H}$. Both methods result in precise quantitation of substrate metabolism. Perhaps the most important consideration when interpreting these results is that embryo metabolism is not only affected by the conditions in which the embryo develops, but also the medium in which metabolism is assessed (Gardner and Leese, 1990; Lane and Gardner, 1998; Krisher et al., 1999; Gandhi et al., 2001). Another drawback to metabolic measurement is that we are unable to measure the metabolic pathways that are normally used by embryos in vivo, so we are never completely confident of what an embryo should be doing metabolically. Of course, we can compare the metabolism of in vivo-derived embryos to that of in vitro cultured embryos, but we must keep in mind that there will likely be some sort of adaptation to the in vitro environment (Lane and Gardner, 1998). Even given these caveats, metabolic studies have provided important information that has helped us understand metabolic mechanisms in mammalian embryos.

\section{Metabolomics}

It is only recently that technological advances in automation and information technology have allowed the basic techniques of metabolomics to be applied to the study of embryo metabolism (Hollywood et al., 2006; Brison et al., 2007; Seli et al., 2007; Krisher et al., 2015). Metabolomics offers multiple advantages over previous methods. This technology is able to measure uptake and production of multiple substrates by the embryo by non-invasively analyzing the medium following in vitro culture. This represents a significant advance in our ability to examine embryo metabolism in a complex environment during preimplantation development, compared to our current snapshots of isolated pathways measured in modified media not designed to support long term embryo culture. This approach also leaves the embryo viable for transfer, thus lending itself to the discovery of a metabolic signature characteristic of high quality embryos that could be used to select embryos for transfer (Singh and Sinclair, 2007).

Typically, medium is analyzed following in vitro embryo culture and compared to medium without an embryo to ascertain how the composition of the culture medium was altered, commonly referred to as the metabolic 'footprint' of the embryo. Although an indirect measurement, it provides specific information about what substrates the embryo is taking up and producing, providing clues as to the pathways in operation. Metabolomics provides information about how the embryos use substrates that we know are included in the culture medium using a targeted approach (measuring a predefined set of metabolites). In addition, a non-targeted approach can be used that will collect information about all detectable metabolites, known and unknown, to generate novel information about embryo biochemistry. While the non-targeted approach investigates a larger cohort of metabolites, the datasets are large and complex. Recent improvements in informatics workflow for metabolomics have helped 
mitigate this issue, improving both metabolite annotation and interpretation on a large scale. Another key point to consider when using metabolomics to study embryo metabolism is that quantitation is usually relative. In this circumstance, the amount of a particular substrate taken up by the embryo is reported as a percentage of what was detected in medium without an embryo. This makes inclusion of appropriate media controls, collected from the same culture dish and treated identically to sample drops, paramount. Although this method of relative quantitation does not provide information about the concentration of any given substrate, it does provide data regarding those substrates embryos are consuming or producing in statistically significant quantities compared to the total amount available. Absolute quantitation is possible for some known metabolites by calibrating sample values to a standard curve, which permits better comparison between metabolomic studies as well as to metabolism studies carried out using other techniques. However, this increases cost and can be difficult to do for large numbers of metabolites. It is important to note that absolute quantitation is not necessary to make valid metabolomic comparisons. Often, both uni- and multivariate statistical analyses enable recognition of differences or changes in metabolite profiles that can be used as markers of disease or toxicity, even before specific metabolites are quantified, or even identified.

Several platforms can be adapted for metabolomics, although mass-spectrometry based approaches are ideally suited for the sensitivity, complex composition and low sample volume inherent in analyses of embryo culture media. Multiple platforms have been reported for analysis of embryo metabolism, including gas or liquid chromatography (GC and LC, respectively) and/or matrix-assisted laser desorption/ionization (MALDI) coupled to mass spectrometry (MS), as well as nuclear magnetic resonance (NMR), Raman, or near infra-red (NIR) spectroscopy. If MS is used, measurement of the molecules' mass, or the masses of distinctive fragments of that molecule following derivitization, results in a specific molecular fingerprint that then allows identification of the metabolite when compared to known databases. The sensitivity of these methods permits the analysis of individual embryos, negating the need for embryo pooling and providing the opportunity to associate specific metabolic profiles with embryo competence post transfer.

Along with the power inherent in the application of metabolomic technology to embryo metabolism, there are some limitations. It is only possible to detect net differences in culture medium with and without an embryo. If the same substrate is both consumed and produced by the embryo, resulting in a net change of zero, it will not be detected as metabolic activity. It is also not possible to differentiate between the same substrate originating from the culture medium or the embryo. For example, these techniques cannot distinguish between lactate from the culture medium and lactate produced by the embryo, only the total lactate value is obtained. Substrates labeled with stable isotopes ( such as ${ }^{13} \mathrm{C}$ ) can be used to overcome these problems, but not in embryos destined for transfer. Similarly, if the culture medium lacks a metabolite important for embryo metabolism and development, a metabolomics approach will not reveal its absence. An additional limitation of metabolomics is that to obtain information about many intermediates in metabolic pathways, which is critical to understanding pathway preference, the embryo must be analyzed directly and thus destroyed. Additionally, in most cases fewer metabolites will be analyzed than are actually detected in the complete sample spectrum. This may be because a targeted approach is used where only known metabolites are specifically examined, because some metabolites are unknown, or because some were not accurately detected. A final drawback to metabolomics technology is that current platforms are expensive and complex, requiring experts to both run the samples and analyze the data, resulting in relatively slow throughput and making them unrealistic for most assisted reproductive technology (ART) laboratories (Montag et al., 2013). However, blastocyst vitrification provides the time necessary to perform these complex analyses at a specialized core facility prior to embryo transfer.

\section{Applications of metabolomic technology to assisted reproduction}

Metabolomic profiling provides a large amount of information describing the metabolic activity of individual embryos. Now that we can successfully undertake such studies, we must consider the impact that this information might have in ART. Can the knowledge generated by this technology improve ART? Certainly, a primary outcome is that of basic knowledge leading to an improved understanding of embryo metabolism. Then we can expand our experiments to determine how embryo metabolism changes during preimplantation development, discover how embryo metabolism is altered by maternal disease, and in what manner embryo quality is reflected by metabolism. Finally, we can then address the overarching question of how these factors interact with the environment in which the embryo finds itself to influence competence. These studies should lead to the formulation of improved culture media that manage embryo metabolism to alter the activities of specific pathways critical to embryo quality that are not supported in conventional media.

Metabolomics has been used for research of embryo metabolism, providing novel basic information. Although analyzed in groups, the metabolome of mouse embryos has been defined using tandem mass spectrometry (LC-MS/MS) and capillary electrophoresis TOF-MS (Wale and Gardner, 2012; Yamada et al., 2012). Our laboratory has reported metabolomic analyses of mouse, bovine and human embryos using GC- and MALDI- MS relative to species, stage of development, embryo quality, maternal characteristics, and culture conditions (Krisher et al., 2015). Lipids are one class of metabolites that have begun to be studied in depth in oocytes and embryos using metabolomic 
techniques. Using MALDI-MS, the lipid content of individual oocytes and embryos from several species was defined, and alterations in lipid profile of bovine embryos due to culture with serum were described (Ferreira et al., 2010). MALDI time of flight (TOF) MS was used to evaluate the lipid profile of human cumulus cells, demonstrating that phosphatidylcholine might be used as a marker of oocytes capable of producing an embryo that results in pregnancy (Montani et al., 2012). Desorption electrospray ionization mass spectrometry (DESI-MS) has been used to describe changes in lipid profile during preimplantation development in the mouse, and described differences between embryos produced in vitro and in vivo (Ferreira et al., 2012). In bovine embryos, MALDI-MS revealed differences in phosphatidylcholine and sphingomyelin due to in vitro culture as well as subspecies of origin (Sudano et al., 2012).

A relatively unheralded application of metabolomics in the ART laboratory is the identification of embryo-toxic contaminants in contact materials. Significant quality testing is currently performed, typically using the mouse embryo assay, to determine the suitability of specific lots of reagents and plastic ware for human embryo culture. Many products are tested by both the supplier and the end user, and products are detected that compromise embryo development. However, there is no understanding of the contaminating compounds present that render lots unsuitable. If these compounds could be identified, products could be prescreened to eliminate those with known contaminants causing negative effects on embryo growth. This would not only significantly reduce the chance that these products would reach the ART laboratory, but would also decrease resources used for testing.

Probably the most anticipated application of metabolomics to ART, however, is the development of a biomarker for embryo viability. Given that embryo metabolism is so closely linked to viability, a metabolic biomarker is of great interest (Nel-Themaat and Nagy, 2011; Gardner and Wale, 2013). Research has provided compelling evidence that metabolism, and amino acid turnover in particular, is related to embryo quality in humans and other mammalian species (Houghton et al., 2002; Brison et al., 2004; Sturmey et al., 2008, 2010; Hemmings et al., 2012; Gardner and Wale, 2013). To date, morphology is the most widely used method by which to identify viable embryos. However, it is widely accepted that this parameter provides only limited information about an embryos ability to implant and support a viable pregnancy (Botros et al., 2008). Initial reports suggested that metabolomic analyses may provide a better predictive tool for embryo selection, compared to morphology alone (Nagy et al., 2008; Marhuenda-Egea et al., 2010; Cortezzi et al., 2013). Retrospective studies using Raman and NIR spectroscopy, as well as electrospray ionization MS (ESI-MS), defined associations between spent media profiles and the potential for successful implantation in human ART (Nagy et al., 2008; Sakkas et al., 2008; Scott et al., 2008; Seli et al., 2010; Marhuenda-Egea et al., 2011; Pudakalakatti et al., 2013; Zivi et al., 2014). Randomized controlled trials based upon these retrospective results were undertaken but selection using the metabolomics based viability index did not increase pregnancy rate compared to selection based upon morphology alone (Hardarson et al., 2012; Vergouw et al., 2012; Uyar and Seli, 2014), possibly due to limitations in sensitivity of this platform (Gardner and Wale, 2013). Of interest, these studies did not identify specific metabolites, only calculated a viability index based upon the spectrum of unspent media. The goal here was biomarker-based prediction, not the generation of knowledge that would inform what we know of embryo metabolism.

\section{Conclusions}

It is clear that metabolic activity is a critical indicator of embryo viability. The success of assisted reproductive technologies involving even a small amount of time in culture is dependent on providing the embryo with an appropriate combination of substrates that will support normal metabolic activity and minimize cellular stress. Although our understanding of embryo metabolism has improved greatly since the early work of Biggers and Brinster (xxx), there is still much work to be done. To understand the relationship between metabolism and viability, we must examine the complex metabolic pathways in total and appreciate their interrelationships. We are just starting to realize the diversity of metabolic mechanisms present among embryos from different species. Previous studies have provided only snapshots of metabolic pathways in isolation. However, application of metabolomic technologies to the analysis of embryo metabolism permits visualization of metabolism in optimized culture conditions and in the context of a complete metabolic system. To date, metabolomic technology has been successfully applied to the study of embryo metabolism, although most studies have been descriptive in nature. These initial studies have provided important new information about the metabolic activity of embryos during development in vitro, and have begun to address the relationship between metabolism and quality. Now the field is poised to expand this work to address experimental hypotheses for basic research, and apply the knowledge gained. Ultimately metabolomic data will provide in depth detail of biochemical pathways used by embryos under various conditions, revolutionizing our understanding of embryo biochemistry and leading to the ability to manipulate metabolism in vitro to support improved embryo development, and allowing the identification of the most metabolically viable embryos.

\section{Acknowledgments}

The authors wish to thank Alison Greene and Chad Barentsen for their assistance with metabolomics experiments in the laboratory that informed the content of this manuscript. Sarah Lyons, Jay Kirkwood, Corey Broeckling, and Jessica Prenni of the Colorado State 
University Proteomics and Metabolomics Core facility have provided invaluable assistance in designing, optimizing, running, troubleshooting and discussing metabolomics platforms for analysis of embryo metabolism.

\section{References}

Baltz JM, Zhou C. 2012. Cell volume regulation in mammalian oocytes and preimplantation embryos. $\mathrm{Mol}$ Reprod Dev, 79:821-831.

Baumann CG, Morris DG, Sreenan JM, Leese HJ. 2007. The quiet embryo hypothesis: molecular characteristics favoring viability. Mol Reprod Dev, 74:1345-1353.

Biggers JD, Whittingham DG, Donahue RP. 1967. The pattern of energy metabolism in the mouse oocyte and zygote. Proc Natl Acad Sci, 58:560-567.

Biggers JD, Mcginnis LK, Raffin M. 2000. Amino acids and preimplantation development of the mouse in protein-free potassium simplex optimized medium. Biol Reprod, 63:281-293.

Botros L, Sakkas D, Seli E. 2008. Metabolomics and its application for non-invasive embryo assessment in IVF. Mol Hum Reprod, 14:679-690.

Brinster RL. 1965a. Studies on the development of mouse embryos in vitro. II. The effect of energy source. J Exp Zool, 158:59-68.

Brinster RL. 1965b. Studies on the development of mouse embryos in vitro. IV. Interaction of energy sources. J Reprod Fertil, 10:227-240.

Brison DR, Houghton FD, Falconer D, Roberts SA, Hawkhead J, Humpherson PG, Lieberman BA, Leese HJ. 2004. Identification of viable embryos in IVF by non-invasive measurement of amino acid turnover. Hum Reprod, 19:2319-2324.

Brison DR, Hollywood K, Arnesen R, Goodacre R 2007. Predicting human embryo viability: the road to non-invasive analysis of the secretome using metabolic footprinting. Reprod Biomed Online, 15:296-302.

Comizzoli P, Urner F, Sakkas D, Renard JP. 2003. Up-regulation of glucose metabolism during male pronucleus formation determines the early onset of the s phase in bovine zygotes. Biol Reprod, 68:1934-1940.

Cortezzi SS, Cabral EC, Trevisan MG, Ferreira CR, Setti AS, Braga DP, Figueira RC, Iaconelli A Jr, Eberlin MN, Borges E Jr. 2013. Prediction of embryo implantation potential by mass spectrometry fingerprinting of the culture medium. Reproduction, 145:453-462.

Dunning KR, Cashman K, Russell DL, Thompson JG, Norman RJ, Robker RL. 2010. Beta-oxidation is essential for mouse oocyte developmental competence and early embryo development. Biol Reprod, 83:909918.

Ferguson EM, Leese HJ. 2006. A potential role for triglyceride as an energy source during bovine oocyte maturation and early embryo development. Mol Reprod Dev, 73:1195-1201.

Ferreira CR, Saraiva SA, Catharino RR, Garcia JS, Gozzo FC, Sanvido GB, Santos LFA, Lo Turco EG, Pontes JHF, Basso AC, Bertolla RP, Sartori R,
Guardieiro MM, Perecin F, Meirelles FV, Sangalli JR, Eberlin MN. 2010. Single embryo and oocyte lipid fingerprinting by mass spectrometry. J Lipid Res, 51:1218-1227.

Ferreira CR, Pirro V, Eberlin LS, Hallett JE, Cooks RG. 2012. Developmental phases of individual mouse preimplantation embryos characterized by lipid signatures using desorption electrospray ionization mass spectrometry. Anal Bioanal Chem, 404:2915-2926.

Gandhi AP, Lane M, Gardner DK, Krisher RL. 2000. A single medium supports development of bovine embryos throughout maturation, fertilization and culture. Hum Reprod, 15:395-401.

Gandhi AP, Lane M, Gardner DK, Krisher RL. 2001. Substrate utilization in porcine embryos cultured in NCSU23 and G1.2/G2.2 sequential culture media. Mol Reprod Dev, 58:269-275.

Gardner DK, Leese HJ. 1986. Non-invasive measurement of nutrient uptake by single cultured preimplantation mouse embryos. Hum Reprod, 1:25-27.

Gardner DK, Leese HJ. 1987. Assessment of embryo viability prior to transfer by the noninvasive measurement of glucose uptake. J Exp Zool, 242:103105.

Gardner DK, Leese HJ. 1988. The role of glucose and pyruvate transport in regulating nutrient utilization by preimplantation mouse embryos. Development, 104:423-429.

Gardner DK, Leese HJ. 1990. Concentrations of nutrients in mouse oviduct fluid and their effects on embryo development and metabolism in vitro. $J$ Reprod Fertil, 88:361-368.

Gardner DK, Lane M, Batt P. 1993. Uptake and metabolism of pyruvate and glucose by individual sheep preattachment embryos developed in vivo. Mol Reprod Dev, 36:313-319.

Gardner DK, Lane M, Stevens J, Schoolcraft WB. 2001. Noninvasive assessment of human embryo nutrient consumption as a measure of developmental potential. Fertil Steril, 76:1175-1180.

Gardner DK, Wale PL. 2013. Analysis of metabolism to select viable human embryos for transfer. Fertil Steril, 99:1062-1072.

Gopichran N, Leese HJ. 2003. Metabolic characterization of the bovine blastocyst, inner cell mass, trophectoderm and blastocoel fluid. Reproduction, 126:299-308

Haggarty P, Wood M, Ferguson E, Hoad G, Srikantharajah A, Milne E, Hamilton M, Bhattacharya S. 2006. Fatty acid metabolism in human preimplantation embryos. Hum Reprod, 21:766-773.

Hardarson T, Ahlstrom A, Rogberg L, Botros L, Hillensjo T, Westlander G, Sakkas D, Wikland M. 2012. Non-invasive metabolomic profiling of day 2 and 5 embryo culture medium: a prospective randomized trial. Hum Reprod, 27:89-96.

Harris SE, Gopichandran N, Picton HM, Leese HJ, Orsi NM. 2005. Nutrient concentrations in murine follicular fluid and the female reproductive tract. Theriogenology, 64:992-1006.

Hasler JF. 2000. In-vitro production of cattle embryos: problems with pregnancy and parturition. Hum Reprod, 
15(suppl.5):47-58.

Hemmings KE, Leese HJ, Picton HM. 2012. Amino acid turnover by bovine oocytes provides an index of oocyte developmental competence in vitro. Biol Reprod, $86: 165,161-112$.

Hewitson LC, Martin KL, Leese HJ. 1996. Effects of metabolic inhibitors on mouse preimplantation embryo development and the energy metabolism of isolated inner cell masses. Mol Reprod Dev, 43:323-330.

Hollywood K, Brison DR, Goodacre R. 2006. Metabolomics: current technologies and future trends. Proteomics, 6: 4716-4723.

Homa ST, Racowsky C, Mcgaughey RW. 1986. Lipid analysis of immature pig oocytes. J Reprod Fertil, 77:425-434

Houghton FD, Thompson JG, Kennedy CJ, Leese HJ. 1996. Oxygen consumption and energy metabolism of the early mouse embryo. Mol Reprod Dev, 44:476485.

Houghton FD, Hawkhead JA, Humpherson PG, Hogg JE, Balen AH, Rutherford AJ, Leese HJ. 2002. Non-invasive amino acid turnover predicts human embryo developmental capacity. Hum Reprod, 17:9991005.

Hugentobler SA, Diskin MG, Leese HJ, Humpherson PG, Watson T, Sreenan JM, Morris DG. 2007. Amino acids in oviduct and uterine fluid and blood plasma during the estrous cycle in the bovine. Mol Reprod Dev, 74:445-454.

Hugentobler SA, Humpherson PG, Leese HJ, Sreenan JM, Morris DG. 2008. Energy substrates in bovine oviduct and uterine fluid and blood plasma during the oestrous cycle. Mol Reprod Dev, 75:496-503. Hugentobler SA, Sreenan JM, Humpherson PG, Leese HJ, Diskin MG, Morris DG. 2010. Effects of changes in the concentration of systemic progesterone on ions, amino acids and energy substrates in cattle oviduct and uterine fluid and blood. Reprod Fertil Dev, 22:684-694.

Humpherson PG, Leese HJ, Sturmey RG. 2005. Amino acid metabolism of the porcine blastocyst. Theriogenology, 64:1852-1866.

Khandoker M, Tsujii H. 1998. Metabolism of exogenous fatty acids by preimplantation rabbit embryos. Japan J Fertil Steril, 43:195-201.

Khurana NK, Niemann H. 2000. Energy metabolism in preimplantation bovine embryos derived in vitro or in vivo. Biol Reprod, 62:847-856.

Kim JY, Kinoshita M, Ohnishi M, Fukui Y. 2001 Lipid and fatty acid analysis of fresh and frozen-thawed immature and in vitro matured bovine oocytes. Reproduction, 122:131-138.

Krisher RL, Lane M, Bavister BD. 1999 Developmental competence and metabolism of bovine embryos cultured in semi-defined and defined culture media. Biol Reprod 60: 1345-1352.

Krisher RL, Prather RS. 2012. A Role for the Warburg Effect in Preimplantation Embryo Development:Metabolic Modification to Support Rapid Cell Proliferation. Mol Reprod Dev, 79: 311-320.

Krisher RL, Heuberger AL, Paczkowski M, Stevens J, Pospisil C, Prather RS, Sturmey RG, Herrick JR,
Schoolcraft WB. 2015. Applying metabolomic analyses to the practice of embryology; physiology, development and ART. Reprod Fertil Dev, 27:602-620.

Lane M, Gardner DK. 1997. Differential regulation of mouse embryo development and viability by amino acids. J Reprod Fertil, 109:153-164.

Lane M, Gardner DK. 1998. Amino acids and vitamins prevent culture-induced metabolic perturbations and associated loss of viability of mouse blastocysts. Hum Reprod, 13:991-997.

Lane M, Hooper K, Gardner DK. 2001. Effect of essential amino acids on mouse embryo viability and ammonium production. J Assist Reprod Genet, 18:519525.

Leese HJ, Barton AM. 1984. Pyruvate and glucose uptake by mouse ova and preimplantation embryos. $J$ Reprod Fertil, 72:9-13.

Leese HJ. 2002. Quiet please, do not disturb: a hypothesis of embryo metabolism and viability. Bioessays, 24:845-849.

Leese HJ, Sturmey RG, Baumann CG, McEvoy TG. 2007. Embryo viability and metabolism: obeying the quiet rules. Hum Reprod, 22:3047-3050.

Leese HJ, Baumann CG, Brison DR, McEvoy TG, Sturmey RG. 2008. Metabolism of the viable mammalian embryo: quietness revisited. Mol Hum Reprod, 14:667-672.

Leroy JLMR, Genicot G, Donnay I, Van Soom A. 2005a. Evaluation of the lipid content in bovine oocytes and embryos with nile red: a practical approach. Reprod Domest Anim, 40:76-78.

Leroy JLMR, Vanholder T, Mateusen B, Christophe A, Opsomer G, De Kruif A, Genicot G, Van Soom A. 2005b. Non-esterified fatty acids in follicular fluid of dairy cows and their effect on developmental capacity of bovine oocytes in vitro. Reproduction, 130:485-495.

Li R, Whitworth K, Lai L, Wax D, Spate L, Murphy CN, Rieke A, Isom C, Hao Y, Zhong Z, Katayama M, Schatten H, Prather RS. 2007. Concentration and composition of free amino acids and osmolalities of porcine oviductal and uterine fluid and their effects on development of porcine IVF embryos. Mol Reprod Dev, 74:1228-1235.

Liu Z, Foote RH. 1995. Effects of amino acids on the development of in-vitro matured/in-vitro fertilization bovine embryos in a simple protein-free medium. Hum Reprod, 10:2985-2991.

Marei WF, Wathes DC, Fouladi-Nashta AA. 2010. Impact of linoleic acid on bovine oocyte maturation and embryo development. Reproduction, 139:979-988.

Marhuenda-Egea FC, Martinez-Sabater E, Gonsalvez-Alvarez R, Lledo B, Ten J, Bernabeu R. 2010. A crucial step in assisted reproduction technology: human embryo selection using metabolomic evaluation. Fertil Steril, 94:772-774.

Marhuenda-Egea F, Gonsálvez-Álvarez R, MartínezSabater E, Lledó B, Ten J, Bernabeu R. 2011. Improving human embryos selection in IVF: noninvasive metabolomic and chemometric approach. Metabolomics, 7:247-256.

Matorras R, Ruiz JI, Mendoza R, Ruiz N, Sanjurjo P, Rodriguez-Escudero FJ. 1998. Fatty acid 
composition of fertilization-failed human oocytes. Hum Reprod, 13:2227-2230.

McEvoy TG, Coull GD, Broadbent PJ, Hutchinson JS, Speake BK. 2000. Fatty acid composition of lipids in immature cattle, pig and sheep oocytes with intact zona pellucida. J Reprod Fertil, 118:163-170.

McKiernan SH, Clayton MK, Bavister BD. 1995. Analysis of stimulatory and inhibitory amino acids for development of hamster one-cell embryos in vitro. $\mathrm{Mol}$ Reprod Dev, 42:188-199.

Montag M, Toth B, Strowitzki T. 2013. New approaches to embryo selection. Reprod Biomed Online, 27:539-546.

Montani DA, Cordeiro FB, Regiani T, Victorino AB, Pilau EJ, Gozzo FC, Ferreira CR, Fraietta R, Lo Turco EG. 2012. The follicular microenviroment as a predictor of pregnancy: MALDI-TOF MS lipid profile in cumulus cells. J Assist Reprod Genet, 29:1289-1297.

Nagy ZP, Sakkas D, Behr B. 2008. Symposium: innovative techniques in human embryo viability assessment. Non-invasive assessment of embryo viability by metabolomic profiling of culture media ('metabolomics'). Reprod Biomed Online, 17:502-507.

Nel-Themaat L, Nagy ZP. 2011. A review of the promises and pitfalls of oocyte and embryo metabolomics. Placenta, 32(suppl.3):S257-263.

O'Fallon JV, Wright RW Jr. 1986. Quantitative determination of the pentose phosphate pathway in preimplantation mouse embryos. Biol Reprod, 34:58-64. Orsi NM, Leese HJ. 2004. Amino acid metabolism of preimplantation bovine embryos cultured with bovine serum albumin or polyvinyl alcohol. Theriogenology, 61:561-572.

Paczkowski M, Schoolcraft WB, Krisher RL. 2014. Fatty acid metabolism during maturation affects glucose uptake and is essential to oocyte competence. Reproduction, 148:429-439.

Panopoulos A, Yanes O, Ruiz S, Kida Y, Diep D, Tautenhahn R, Herrerã-As AD, Batchelder E, Plongthongkum N, Lutz M, Berggren WT, Zhang K, Evans R, Siuzdak G, Izpisua Belmonte J. 2012. The metabolome of induced pluripotent stem cells reveals metabolic changes occurring in somatic cell reprogramming. Cell Res, 22:168-177.

Pantaleon M, Ryan JP, Gil M, Kaye PL. 2001. An unusual subcellular localization of GLUT1 and link with metabolism in oocytes and preimplantation mouse embryos. Biol Reprod, 64:1247-1254.

Petters RM, Johnson BH, Reed ML, Archibong AE. 1990. Glucose, glutamine and inorganic phosphate in early development of the pig embryo in vitro. $J$ Reprod Fertil, 89:269-275.

Pudakalakatti SM, Uppangala S, D'Souza F, Kalthur G, Kumar P, Adiga SK, Atreya HS. 2013. NMR studies of preimplantation embryo metabolism in human assisted reproductive techniques: a new biomarker for assessment of embryo implantation potential. NMR Biomed, 26:20-27.

Rieger D, Loskutoff NM, Betteridge KJ. 1992. Developmentally related changes in the uptake and metabolism of glucose, glutamine and pyruvate by cattle embryos produced in vitro. Reprod Fertil Dev, 4:547-
557.

Rizos D, Ward F, Duffy P, Boland MP, Lonergan P. 2002. Consequences of bovine oocyte maturation, fertilization or early embryo development in vitro versus in vivo: Implications for blastocyst yield and blastocyst quality. Mol Reprod Dev, 61:234-248.

Rosenkrans CF Jr, First NL. 1994. Effect of free amino acids and vitamins on cleavage and developmental rate of bovine zygotes in vitro. $J$ Anim Sci, 72:434-437.

Saito T, Hiroi M, Kato T. 1994. Development of glucose utilization studied in single oocytes and preimplantation embryos from mice. Biol Reprod 50:266-270.

Sakkas D, Morita H, Yamashita N, Kato O, Botros L, Roos P, Seli E. 2008. Evaluation of embryo quality by metabolomics: a new strategy to aid single embryo transfer. J Mamm Ova Res, 25:26-31.

Scott R, Seli E, Miller K, Sakkas D, Scott K, Burns DH. 2008. Noninvasive metabolomic profiling of human embryo culture media using Raman spectroscopy predicts embryonic reproductive potential: a prospective blinded pilot study. Fertil Steril, 90:77-83. Seli E, Sakkas D, Scott R, Kwok SC, Rosendahl SM, Burns DH. 2007. Noninvasive metabolomic profiling of embryo culture media using Raman and near-infrared spectroscopy correlates with reproductive potential of embryos in women undergoing in vitro fertilization. Fertil Steril, 88:1350-1357.

Seli E, Vergouw CG, Morita H, Botros L, Roos P, Lambalk CB, Yamashita N, Kato O, Sakkas D. 2010. Noninvasive metabolomic profiling as an adjunct to morphology for noninvasive embryo assessment in women undergoing single embryo transfer. Fertil Steril, 94:535-542.

Singh R, Sinclair KD. 2007. Metabolomics: approaches to assessing oocyte and embryo quality. Theriogenology, 68(suppl.1):S56-62.

Smith DG, Sturmey RG. 2013. Parallels between embryo and cancer cell metabolism. Biochem Soc Trans, 41:664-669.

Somfai T, Kaneda M, Akagi S, Watanabe S, Haraguchi S, Mizutani E, Dang-Nguyen TQ, Geshi M, Kikuchi K, Nagai T. 2011. Enhancement of lipid metabolism with L-carnitine during in vitro maturation improves nuclear maturation and cleavage ability of follicular porcine oocytes. Reprod Fertil Dev, 23:912920.

Spindler RE, Pukazhenthi BS, Wildt DE. 2000. Oocyte metabolism predicts the development of cat embryos to blastocyst in vitro. Mol Reprod Dev, 56:163171.

Steeves TE, Gardner DK. 1999. Temporal and differential effects of amino acids on bovine embryo development in culture. Biol Reprod, 61:731-740.

Sturmey RG, Brison DR, Leese HJ. 2008. Symposium: innovative techniques in human embryo viability assessment. Assessing embryo viability by measurement of amino acid turnover. Reprod BioMed Online, 17:486-496.

Sturmey RG, Bermejo-Alvarez P, Gutierrez-Adan A, Rizos D, Leese HJ, Lonergan P. 2010. Amino acid 
metabolism of bovine blastocysts: a biomarker of sex and viability.[Erratum appears in Mol Reprod Dev, 77:472, 2010]. Mol Reprod Dev, 77:285-296.

Sudano MJ, Santos VG, Tata A, Ferreira CR, Paschoal DM, Machado R, Buratini J, Eberlin MN, Landim-Alvarenga FD. 2012. Phosphatidylcholine and sphingomyelin profiles vary in Bos taurus indicus and Bos taurus taurus in vitro- and in vivo-produced blastocysts. Biol Reprod, 87:130.

Sutton-McDowall ML, Feil D, Robker RL, Thompson JG, Dunning KR. 2012. Utilization of endogenous fatty acid stores for energy production in bovine preimplantation embryos. Theriogenology, 77:1632-1641.

Suzuki C, Yoshioka K. 2006. Effects of amino acid supplements and replacement of polyvinyl alcohol with bovine serum albumin in porcine zygote medium. Reprod Fertil Dev, 18:789-795.

Takahashi Y, First NL. 1992. In vitro development of bovine one cell embryos: influence of glucose, lactate, pyruvate, amino acids and vitamins. Theriogenology, 37:963-978.

Tervit HR, Whittingham DG, Rowson LEA. 1972. Successful culture in vitro of sheep and cattle ova. $J$ Reprod Fertil, 30:493-497.

Thompson JG, Simpson AC, Pugh PA, Wright RW Jr, Tervit HR. 1991. Glucose utilization by sheep embryos derived in vivo and in vitro. Reprod Fertil Dev, 3:571-576.

Thompson JG, Simpson AC, Pugh PA, Tervit HR. 1992. Requirement for glucose during in vitro culture of sheep preimplantation embryos. Mol Reprod Dev, 31:253-257.

Thompson JG, Bell AC, Pugh PA, Tervit HR. 1993 Metabolism of pyruvate by pre-elongation sheep embryos and effect of pyruvate and lactate concentrations during culture in vitro. Reprod Fertil Dev, 5:417-423.

Thompson JG, Partridge RJ, Houghton FD, Cox CI, Leese HJ. 1996. Oxygen uptake and carbohydrate metabolism by in vitro derived bovine embryos. $J$ Reprod Fertil, 106:299-306.

Uyar A, Seli E. 2014. Metabolomic assessment of embryo viability. Semin Reprod Med, 32:141-152.

Van Hoeck V, Sturmey RG, Bermejo-Alvarez P, Rizos D, Gutierrez-Adan A, Leese HJ, Bols PEJ, Leroy J. 2011. Elevated non-esterified fatty acid concentrations during bovine oocyte maturation compromise early embryo physiology. PloS One, 6:e23183.
Vander Heiden MG, Cantley LC, Thompson CB. 2009. Understanding the Warburg effect: the metabolic requirements of cell proliferation. Science, 324:10291033.

Vander Heiden MG, Locasale JW, Swanson KD, Sharfi H, Heffron GJ, Amador-Noguez D, Christofk HR, Wagner G, Rabinowitz JD, Asara JM, Cantley LC. 2010. Evidence for an alternative glycolytic pathway in rapidly proliferating cells. Science, 329:1492-1499.

Vergouw CG, Kieslinger DC, Kostelijk EH, Botros LL, Schats R, Hompes PG, Sakkas D, Lambalk CB. 2012. Day 3 embryo selection by metabolomic profiling of culture medium with near-infrared spectroscopy as an adjunct to morphology: a randomized controlled trial. Hum Reprod, 27:2304-2311.

Wale PL, Gardner DK. 2012. Oxygen regulates amino acid turnover and carbohydrate uptake during the preimplantation period of mouse embryo development. Biol Reprod, 87:24, 21-28.

Wale PL, Gardner DK. 2013. Oxygen affects the ability of mouse blastocysts to regulate ammonium. Biol Reprod, 89:75.

Wales RG, Brinster RL. 1968. The uptake of hexoses by pre-implantation mouse embryos in vitro. $J$ Reprod Fertil, 15:415-422.

Warburg O. 1956. On the origin of cancer cells. Science, 123:309-314.

Whitten WK, Biggers JD. 1968. Complete development in vitro of the pre-implantation stages of the mouse in a simple chemically defined medium. $J$ Reprod Fertil, 17:399-401.

Wu GQ, Jia BY, Li JJ, Fu XW, Zhou GB, Hou YP, Zhu SE. 2011. L-carnitine enhances oocyte maturation and development of parthenogenetic embryos in pigs. Theriogenology, 76:785-793.

Yamada M, Takanashi K, Hamatani T, Hirayama A, Akutsu H, Fukunaga T, Ogawa S, Sugawara K, Shinoda K, Soga T, Umezawa A, Kuji N, Yoshimura Y, Tomita M. 2012. A medium-chain fatty acid as an alternative energy source in mouse preimplantation development. Sci Rep, 2:930.

Zhang J, Nuebel E, Daley G, Koehler C, Teitell M. 2012. Metabolic regulation in pluripotent stem cells during reprogramming and self-renewal. Cell Stem Cell, 11:589-595.

Zivi E, Barash D, Aizenman E, Gibson D, Shufaro Y. 2014. Zygote serine decreased uptake from the fertilization medium is associated with implantation and pregnancy. J Assist Reprod Genet, 31:889-897. 